\title{
Abstracts of scientific presentations at the 2019 National Annual Congress of the Critical Care Society of Southern Africa in Cape Town, South Africa
}

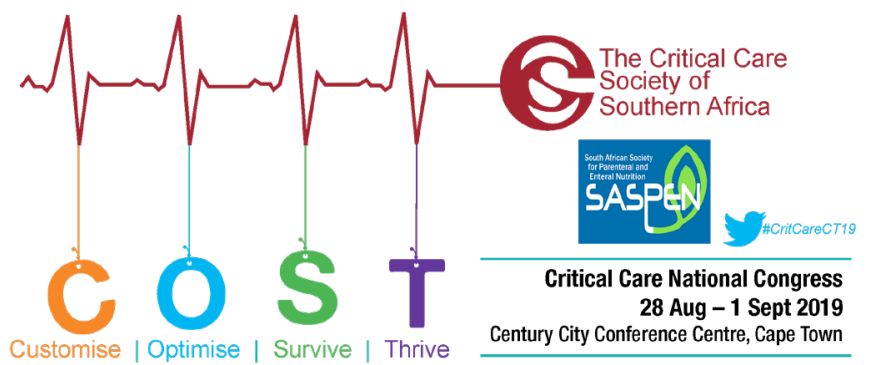

\section{High-frequency oscillatory ventilation in a tertiary ICU in an academic hospital in Johannesburg S Cawood, B Rae, K Naidoo \\ Chris Hani Baragwanath Academic Hospital, Johannesburg, South Africa shancawood@gmail.com}

Background. High-frequency oscillatory ventilation (HFOV) remains an option for the management of critically ill children when conventional mechanical ventilation fails. However, its use is not widespread and there is wide variability in practice.

Objective. To describe HFOV use among paediatric patients with a primary respiratory disorder admitted to a tertiary paediatric intensive care unit. Method. Two-year, single centre, retrospective chart review.

Results. Thirty-four (32.7\%) patients were managed with HFOV with all patients fulfilling criteria for paediatric acute respiratory distress syndrome. Indications for HFOV were inadequate oxygenation in 17 patients (50\%) and refractory respiratory acidosis in 15 patients (44.1\%). Setting of HFOV varied considerably, particularly with respect to initial Paw. HFOV was effective at improving both oxygenation with a median decrease in OI of 6.3 (IQR 4.5; 9.5), and ventilation with a median decrease in $\mathrm{PaCO}_{2}$ of $67.6 \mathrm{~mm} \mathrm{Hg}$ (IQR 46.2; 105.7) after 24 hours. The overall mortality was $29.4 \%$, consistent with other studies but higher than that predicted by paediatric index of mortality 3 (PIM3). Conclusion. HFOV remains an effective rescue ventilatory strategy that results in rapid and sustained improvement in gas exchange in patients with severe hypoxaemia and/or severe respiratory acidosis. However, the variability in practice highlights the need for updated guidelines on HFOV use

\section{Extracranial healthcare-associated complications in severe traumatic brain injury patients at Groote Schuur Hospital's neurocritical care unit C Arnold-Day, A Oseni, P Semple \\ Department of Neurosurgery, Groote Schuur Hospital and Faculty of Health Sciences, University of Cape Town, South Africa \\ patrick.semple@uct.ac.za}

Background. Critically-ill patients are subject to many complications associated with advanced and invasive interventions required to monitor and manage their acute illnesses. Many complications are 'healthcareassociated' and related to invasive monitoring and organ support devices. In the setting of a neurosurgical intensive care unit (NSICU), where patients are being admitted after sustaining severe and lifethreatening brain injuries, these complications impact the patients' prognosis and length of hospital stay.

Objective. To evaluate the most common extracranial, healthcareassociated complications that occur in patients admitted to our NSICU with severe traumatic brain injury over a 22 -month period.

Method. A retrospective review of the Neurocritical Care Database considering all extracranial healthcare-associated complications in all patients with severe traumatic brain injury (GCS $\leq 8)$ admitted to the NSICU from 1 January 2014 - 31 October 2015, requiring ventilatory support.

Results. Of the 282 trauma patients admitted to the NSICU, the most common extracranial, healthcare-associated complications included ventilator associated pneumonia (VAP), hospital acquired pneumonia (HAP), aspiration pneumonitis, cellulitis and acute kidney injury. A subgroup analysis showed a marked decline of the VAP incidence from 2014 to 2015 after our unit implemented the VAP-care bundle and stricter protocolised contact precautions. These results are in keeping with international literature affirming that traumatic brain injury is an important predictor for complications.

Conclusion. Extracranial healthcare-associated complications after traumatic brain injury are common in this extremely vulnerable patient population. These complications are amenable to pre-emptive strategies for prevention, leading to improved patient outcomes.

\section{The utility of hyperlactataemia} in the definition of septic shock: Evaluating the Sepsis-3 definitions in a Sub-Saharan African intensive care unit

\section{A Elhouni}

University of KwaZulu-Natal and Inkosi Albert Luthuli Central Hospital, Durban, South Africa

ali.el_houni@yahoo.com

Background. Sepsis-3 definitions were published in 2016, and included hyperlactataemia (serum lactate $>2.0 \mathrm{mmol} / \mathrm{L}$ ) as a mandatory component of the new definition of septic shock. The data were collected predominantly from high-income countries (HICs), and lack adequate validation in scenarios outside these countries.

Methods. This study was a retrospective observational study of 170 patients with infection and hypotension requiring inotropic support admitted to the intensive care unit at King Edward VIII hospital in South Africa. Admission serum lactate was evaluated as a predictor of intensive care unit (ICU) mortality in this cohort. 
Results. The study population had a median age of only 42 years. The ICU mortality rate for the cohort was $49.4 \%$. Most patients were surgical (71.8\%), with the most common source of sepsis being abdominal (55.9\%). The ICU mortality rate was $40.9 \%$ in those with a lactate of $\leq 2.0$ $\mathrm{mmol} / \mathrm{L}$ and $52.4 \%$ with a lactate of $>2.0 \mathrm{mmol} / \mathrm{L}$, but this did not reach statistical significance. The optimal cut-off was $4.5 \mathrm{mmol} / \mathrm{L}$. At a cut-off of $4.5 \mathrm{mmol} / \mathrm{L}$ there was a clear, statistically significant, difference in mortality between those without hyperlactataemia (39.3\%) and those with hyperlactataemia (59.3\%) $(p=0.009)$.

Conclusion. Hyperlactataemia was associated with increased mortality. However, hyperlactatemia of $>2.0 \mathrm{mmol} / \mathrm{L}$, as proposed in Sepsis-3, did not reach statistical significance. A higher cut-off of $>4.5 \mathrm{mmol} / \mathrm{L}$ was more appropriate than the cut-off of $2.0 \mathrm{mmol} / \mathrm{L}$ suggested in Sepsis-3.

\section{Vitamin D levels in ICU patients and the association with organ dysfunction and glutamine levels F Seedat, ${ }^{1}$ G Schleicher, ${ }^{1}$ R Blaauw ${ }^{2}$ \\ ${ }^{1}$ Wits Donald Gordon Medical Centre, Johannesburg, South Africa ${ }^{2}$ Division of Human Nutrition, University of Stellenbosch, South Africa seedat.faheem@gmail.com}

Background. Vitamin D deficiency is common in ICU patients (50\% $82 \%)$ and is associated with multiorgan dysfunction. Vitamin D status alters glutamine pathways in critical illness, but this relationship is poorly characterised.

Objective. To assess the prevalence of vitamin D deficiency and its association with organ dysfunction and glutamine levels in a South African ICU.

Methods. Records of 103 patients admitted to the WDGMC ICU were retrospectively reviewed. A total of $25-(\mathrm{OH})$ vitamin $\mathrm{D}$ levels at admission and following 7 days of supplementation, admission glutamine levels, SOFA scores and organ support were analysed.

Results. On admission 66\% $(n=68 / 103)$ were vitamin $\mathrm{D}$ deficient $(<20 \mathrm{ng} / \mathrm{mL})$. Of those with day 7 levels $(n=57), 47 \%$ remained deficient despite supplementation. Deficiency was significantly associated with mechanical ventilation $(40 \%$ v. $14 \%)(p=0.013)$ and a higher SOFA score $(6(3-8)$ v. $4(2-6) ; 95 \%$ CI $)(p=0.047)$. Median serum glutamine levels were $480.7 \mu \mathrm{mol} / \mathrm{L}$, with $37.9 \%$ deficient $(<420 \mu \mathrm{mol} / \mathrm{L})$. Vitamin D and glutamine levels showed no significant association $(p=0.66)$.

Conclusion. Vitamin D deficiency is common and persists despite supplementation. Deficient patients were more severely ill and required more respiratory support. No significant relationship between deficiency and glutamine levels was noted.

\section{Influence of staff shortage on work environment: Critical care nurses' experiences \\ M Nakweenda, ${ }^{1}$ R Anthonie, ${ }^{2}$ M Van Der Heever ${ }^{2}$ \\ ${ }^{1}$ School of Nursing, University of Namibia, Namibia \\ ${ }^{2}$ Department of Nursing and Midwifery, Stellenbosch University, South Africa mnakweenda@unam.na}

Background. The global shortage of critical care nurses has contributed to the high workload experienced by these nurses. As a result, critical care nurses have negative experiences related to their work environment.
Objective. The aim of the study was to explore and describe the experiences of critical care nurses regarding the influence of staff shortages on their work environment.

Methods. A descriptive qualitative design was applied. A sample size of 11 was drawn from a total population of 18, using purposive sampling. Data were collected by using a validated semi-structured interview guide, and data saturation was reached by the 11th participant. Data were analysed manually using iterative thematic analysis, according to the following steps: transcription, immersion in data, data reduction, coding, reflection, identification of themes and interpretation. Trustworthiness was assured by adhering to Lincoln and Guba's criteria of credibility, confirmability, transferability and dependability.

Results. Five main themes emerged from the study, namely: working conditions, physical and social environment, resource availability, leadership support and incentives.

Conclusion. It appeared that critical care nurses had to work in compromising conditions characterised by long working hours, insufficient space and equipment, lack of support from managers and poor incentives.

\section{Association between antibiotic trough concentrations and estimated total renal clearance in critically ill patients receiving renal replacement therapy: A pragmatic, multi-national observational study \\ G Joynt, ${ }^{1,2}$ G Choi, ${ }^{1}$ J Roberts, ${ }^{2}$ J Lipman, ${ }^{1,2}$ A Lee, ${ }^{1}$ SMARRT Study Group \\ ${ }^{1}$ Department of Anaesthesia and Intensive Care, The Chinese University \\ of Hong Kong, Shatin, Hong Kong \\ ${ }^{2}$ Burns Trauma and Critical Care Research Centre, University of \\ Queensland, Australia \\ gavinmjoynt@cuhk.edu.hk}

Background. Achieving appropriate blood antibiotic concentrations is important to optimise the treatment of sepsis and improve mortality. Objective. To test the hypothesis that estimated total renal clearance (eTRCL) is inversely associated with trough concentrations achieved in ICU patients receiving continuous renal replacement therapy (CRRT).

Method. Prospective, observational, multi-national, pharmacokinetic study in 26 ICUs. Antibiotic dosing and CRRT prescription were determined by individual unit practice. Patient demographic, CRRT dose, antibiotic dose, clinical data and trough concentrations of meropenem, piperacillin-tazobactam, and vancomycin were evaluated. eTRCL was defined as CRRT clearance (effluent flow rate) plus measured creatinine clearance in patients with urine output.

Results. A total of 384 patients were enrolled from 26 ICUs. Patients received meropenem $(n=187)$, piperacillin $(n=160)$, tazobactam $(n=101)$ and vancomycin $(n=60)$. Generalised estimating equations were used to account for centre-effects. Despite wide variations in antibiotic dose (4 - 8-fold) and CRRT dose achieved (effluent flow rate interquartile range (IQR) $34-58 \mathrm{~mL} / \mathrm{min}$ ) that resulted in a wide range of trough concentrations, there was a consistent association between increasing eTRCL and decreased trough concentration for piperacillin, tazobactam, and vancomycin $(p<0.05)$.

Conclusion. Future antibiotic dosing algorithms to improve the adequacy and consistency of blood antibiotic concentrations in critically ill patients requiring CRRT should take eTRC into account. 


\author{
Streamlining the treatment of cardiac \\ arrest after cardiac surgery \\ J van der Merwe, ${ }^{1} \mathrm{R}$ Verbeek, ${ }^{1}$ A Levine, ${ }^{2}$ Joel Dunning ${ }^{2}$ \\ ${ }^{1}$ Cardia Surgical Unit Advanced Life Support (CSU-ALS), South Africal \\ Atlantic Cardiovascular and Thoracic Institute, Netcare Blaauwberg \\ Hospital, Cape Town, South Africa \\ ${ }^{2}$ James Cook University Hospital, Department of Cardiothoracic Surgery, \\ Middlesbrough, United Kingdom \\ drjohan@acvti.co.za
}

Background. Cardiac arrest after cardiac surgery (CACS) is associated with poor outcomes, and presents unique challenges to the cardiac critical care team.

Objective. It is our intention to introduce recently published internationally endorsed CACS resuscitation protocols to South African critical care providers and to provide a training platform from which these principles can be incorporated into routine cardiac critical care.

Methods. Evidence to create international CACS protocols were obtained from existing guidelines, the International Liaison Committee on Resuscitation, international online surveys and from MEDLINE keyword searches for human clinical studies, animal models and simulation protocols relevant to CACS.

Results. In total, 56 publications were identified to create CACS recommendations. Internal massage (IM) is more effective than external massage (EM) in CACS and should be used preferentially if reversible causes are excluded. External massage (EM) is ineffective in tamponade or hypovolemia and only salvageable by emergency re-sternotomy (ER) within 5 minutes. CACS arrhythmias require unique interventions, as does the administration of resuscitation medication including Epinephrine.

Conclusion. CACS requires unique skillsets, an efficient critical care team with allocated key roles and protocols that differ from commonly used advance cardiac life support guidelines. CACS can potentially be prevented and effectively treated by applying specialised protocols and principles.

\section{The liberation of ventilated adult patients in public critical care units: An intervention study \\ B R Oamen, ${ }^{1}$ P Jordan, ${ }^{2}$ W ten Ham-Baloyi, ${ }^{1}$ E $Z$ Hlungwane ${ }^{1}$ \\ ${ }^{1}$ Nelson Mandela University, Port Elizabeth, South Africa \\ ${ }^{2}$ Stellenbosch University, Cape Town, South Africa \\ benoamen@gmail.com}

Background. The knowledge of critical care nurses (CCNs) about the liberation of ventilated adult patients, especially in the Eastern Cape Province, remains unknown and needs to be addressed to optimise liberation practices.

Objectives. To investigate the knowledge of CCNs on liberation of adult patients and to implement the evidence-based guidelines (EBGs) on liberation using an educational intervention, as well as to describe its effect on their knowledge.

Method. An intervention study following a quasi-experimental design using a pre-test/post-test with a control group was conducted among CCNs (pre-test, $n 1=115$; post-test, $n 2=90$ ). Implementation of an educational intervention in Intervention Group One (including a formal lecture, printed copies of the EBGs, posters and monitoring visits) and Intervention Group Two (including printed copies of the EBGs) was done. Results. There was an increase (mean difference $=-2.28 ; \mathrm{t}=-0.90$; $p=0.37$ ) in mean knowledge score in Intervention Group One, although not significant. However, a significant (mean difference $=6.94 ; t=2.17$; $p=0.03$; Cohen's $d=0.49$ ) decline in mean knowledge score in Intervention Group Two. There was a significant $(\mathrm{F}=5.66 ; p=0.018$; Cohen's $d=0.67$ ) mean difference between $\mathrm{CCNs}$ who had 5 years' or more work experience in critical care units and knowledge score increase.

Conclusion. Participants lacked current knowledge of liberation practices. Knowledge can be enhanced through the presentation of combined educational intervention methods.

\section{Exploring adult patients' perceptions of what enables them to make sense of their intensive care experience} K Harmuth, J Bell

Stellenbosch University, Cape Town, South Africa

kharmuth@sun.ac.za

Background. Critical illness requiring admission into an intensive care environment is a significant stressful event in any person's life.

Objective. To explore adult patients' perceptions of what in their intensive care encounter enabled them to make sense of and in turn move through their experience.

Method. A qualitative descriptive phenomenological research approach was used.

Results. Participants were enabled to make sense of their intensive care experience when they were part of a trusting relationship with their caregiver, allowing them to feel at ease and in turn feel safe. When patients develop distrust in staff ability to competently care for them, their feelings of safety and security are eroded.

Conclusion. The study aims to contribute to understanding what a patient moving through an intensive care experience endures and in turn offer ways to better adapt care and interventions to support a more tolerable experience. The study aims to start conversation about how caregivers can connect with patients in their most vulnerable time of need with emphasis on how the little things matter.

\section{Modified early warning score as a predictor for intensive care unit admission in chemotherapy receiving oncology patients with positive blood culture}

T Felemban, ${ }^{1,2}$ J Allarakia, ${ }^{1,2,3}$ A Alghamdi, ${ }^{1,2,3}$ A Ashi, ${ }^{1,2,3}$

A Alsahafi, ${ }^{1,2,3}$ M Alzahrani, ${ }^{3}$ A Alamri, ${ }^{1,2,3}$ M AlDabbagh,,${ }^{1,2,3}$

${ }^{1}$ King Saud bin Abdulaziz University for Health Sciences, Jeddah, Saudi Arabia

${ }^{2}$ King Abdullah International Medical Research Center, Jeddah, Saudi Arabia

${ }^{3}$ National Guard Health Affairs King Abdulaziz Medical City, Jeddah,

Saudi Arabia

Felemban.ta@gmail.com

Background. Chemotherapy receiving oncology patients (CROP) are prone to sepsis; however, their suppressed immune system renders the signs of inflammation less evident. The Modified Early Warning Score (MEWS) is a tool intended to detect patients with deteriorating clinical circumstances. Objective. To assess the usefulness of MEWS in predicting ICU admission CROP with positive blood culture.

Method. Adults with positive blood cultures were subdivided into two groups: CROP cases and immunocompetent control group; comparison was referenced to the actual ICU admittance. MEWS was calculated at different intervals to identify its discriminative capability. Receiver operator 
curves (ROC) analysis was used to determine the best Cut-off MEWS. Results. From 192 subjects with positive blood culture, 89 CROPs and 103 controls were identified. The sensitivity and specificity for a MEWS of $\geq 4$ in CROP was $52.5 \%$ and $70 \%$, respectively, and this was comparable with the control group. ROC showed that MEWS was a significant predictor for ICU admission if calculated 12 - 36 hours before culture in CROP, and a threshold of $\geq 3$ had the best specificity (86 91\%) for predicting ICU admission.

Conclusion. MEWS has a weak discriminatory value in predicting ICU admission in CROP. A threshold of $\geq 3$ MEWS at $12-36$ hours' culture was found to be the best cut-off for ICU admission prediction. Combining MEWS with clinical judgement might improve ICU admission prediction.

\section{Early identification and referral of organ donors in five private hospitals: a survey to determine the knowledge and views of critical care professional nurses pre and post a PowerPoint training intervention}

\section{Prins, L Human}

Transplant Division, Netcare Christiaan Barnard Memorial Hospital, Cape Town, South Africa

lettie.prins@netcare.co.za

Background. Internationally, the declining number of organ donors does not meet the rising demand for life-saving solid organ transplantation.

Objective. To describe the knowledge and views of critical care professional nurses concerning the early identification and referral of organ donors.

Method. A mixed method, experimental, exploratory, descriptive study design was followed.

Results. Critical care professional nurses $(n=173 / 218)$ participated in the study. The median (interquartile range) score for all participants increased from $60.0 \%$ (48 - 76\%) prior to a PowerPoint training intervention to $96 \%$ (88 - 96\%) after the intervention $(p<0.0001)$. Three main themes emerged from the qualitative questions: stress in dealing with the family and the donor (62\%); inadequate collaboration on donor referral between doctors and nurses (34\%); and the need for a donor referral policy (9\%).

Conclusion. Critical care professional nurses' knowledge regarding early identification and referral of potential organ donors increased significantly following a targeted training intervention. However, support is required to effectively communicate with the donor's family and medical staff.

\section{Adequacy of oral intake in a private intensive care unit in Gauteng province}

\section{A Kloppers, ${ }^{1} \mathrm{R}$ Nel, ${ }^{2} \mathrm{~L}$ van den Berg $^{2}$}

${ }^{1}$ Alta Kloppers and Associates, Alberton, Gauteng, South Africa

${ }^{2}$ Department of Nutrition and Dietetics, Faculty of Health Sciences,

University of the Free State, Bloemfontein, South Africa

alta@dieticianonline.co.za

Background. The prevalence of disease-related malnutrition in acute care facilities is a common phenomenon that negatively affects patient outcomes and treatment costs.

Objective. To assess the adequacy of energy and protein intakes of orally fed patients admitted to the intensive care unit (ICU).
Method. An observational cross-sectional study was conducted. Total energy and protein intakes of 26 participants were assessed and compared with requirements.

Results. Those not receiving oral nutritional supplements (ONS) met $98 \%$ of energy and $68 \%$ of protein requirements. The nutritionally vulnerable group that received ONS met $57.2 \%$ of energy and $53.7 \%$ of protein requirements without ONS, and $76.4 \%$ of energy and $74.3 \%$ of protein requirements with ONS $(p<0.05)$. When divided per body mass index (BMI), the subgroup BMI $<30 \mathrm{~kg} / \mathrm{m}^{2}$ $(n=19)$ had inadequate median intake for energy and protein, with and without ONS. For the subgroup BMI $\geq 30 \mathrm{~kg} / \mathrm{m}^{2}(n=7)$ energy intake exceeded requirements $(125.6 \%)$, while protein intake was inadequate (64.1\%).

Conclusion. Oral intakes were inadequate, particularly in terms of protein. Specific considerations to optimise protein delivery without exceeding energy requirements in the critically ill obese patient are necessary.

\section{High-flow oxygen therapy $v$. standard care in infants with viral bronchiolitis}

S Murphy, E Bruckmann, L Doedens, A Khan, A Salloo, S Omar Intensive Care Unit, Chris Hani Baragwanath Academic Hospital; and University of the Witwatersrand, Johannesburg, South Africa

Susan.Murphy@wits.ac.za

Background. High-flow humidified oxygen therapy has demonstrated benefit in infants with viral bronchiolitis.

Objective. To investigate the efficacy of high-flow humidified oxygen in infants with moderate-severe viral bronchiolitis, when used outside the paediatric ICU, in a hospital with limited PICU resources.

Methods. An RCT, which enrolled 28 infants between 1 month and 2 years of age, with a clinical diagnosis of acute viral bronchiolitis and moderate-severe respiratory distress. Participants were randomised to receive high flow oxygen $2 \mathrm{~L} / \mathrm{kg} / \mathrm{min}$ or oxygen by nasal cannula/mask. Respiratory rate, heart rate, saturations, and Tal score were measured at baseline, $60-90 \mathrm{~min}$ after starting therapy and at $6-12$ hourly intervals. The primary outcome was improvement in respiratory distress (Tal score). A secondary outcome was the need for intubation and ventilation

Results. We demonstrated a significant improvement in the work of breathing (Tal score) in infants who received high-flow oxygen therapy. Additionally, there was also a rapid cardiovascular stabilising effect in the high-flow group. There was a trend to a lower intubation rates in the high-flow group.

Conclusion. High-flow humidified oxygen is a beneficial therapy for infants with moderate-severe viral bronchiolitis, and could potentially reduce the need for intubation and admission to PICU.

\section{Plasma glutamine levels in relation to ICU patient outcomes}

R Blaauw, ${ }^{1}$ D G Nel, ${ }^{2}$ G K Schleicher ${ }^{3}$

${ }^{1}$ Division of Human Nutrition, Stellenbosch University, Cape Town, South Africa

${ }^{2}$ Centre for Statistical Consultation, Stellenbosch University, Cape Town, South Africa

${ }^{3}$ Wits Donald Gordon Medical Centre ICU, Parktown, South Africa rb@sun.ac.za 
Background. Both low and high plasma glutamine levels are associated with increased mortality.

Objective. This study aimed to measure glutamine levels in ICU patients and identify proxy indicators of abnormal glutamine levels.

Methods. Patients admitted to three ICUs in South Africa were enrolled, provided they had not received prior glutamine supplementation. Clinical and biochemical data were collected. Plasma glutamine categories used were: low $(<420 \mu \mathrm{mol} / \mathrm{L})$, normal $(420-700 \mu \mathrm{mol} / \mathrm{L})$, high $(>700 \mu \mathrm{mol} / \mathrm{L})$.

Results. A total of 330 patients (age 47.42 (16.56) years; 56.4\% male) were enrolled (mean APACHE II score 18.57 (8.55) and SOFA score 7.05 (3.78)). On admission, 58.5\% had low (median $299.51 \mu \mathrm{mol} / \mathrm{L}$ ) and $14.2 \%$ high (median $898.87 \mu \mathrm{mol} / \mathrm{L}$ ) plasma glutamine levels. Low plasma glutamine was associated with higher APACHE scores $(p=0.003)$, SOFA scores $(p=0.003)$, CRP values $(p<0.001)$, serum urea $(p=0.008)$ and serum creatinine $(p=0.023)$, and lower serum albumin $(p<0.001)$. The best predictors of low plasma glutamine were admission APACHE score (OR 1.032; $p=0.018$ ), SOFA score (OR 1.077; $p=0.016$ ) and CRP (OR 1.006; $p<0.001$ ). ROC curve analysis revealed a CRP threshold value of $87.95 \mathrm{mg} / \mathrm{L}$ to be indicative of low plasma glutamine levels (AUC 0.7; $p<0.001$ ).

Conclusion. A total of $59 \%$ of ICU patients had low plasma glutamine on admission. Markers of infection and disease severity were significant indicators of low plasma glutamine.

\section{Plasma glutamine and clinical outcome among critically ill children}

\section{J Wilson, ${ }^{1}$ R Blaauw, ${ }^{1}$ B M Morrow, ${ }^{2}$ D G Nel ${ }^{3}$}

${ }^{1}$ Division of Human Nutrition, Stellenbosch University, Cape Town, South Africa

${ }^{2}$ Department of Paediatrics, University of Cape Town, Cape Town, South Africa ${ }^{3}$ Centre for Statistical Consultation, Stellenbosch University, South Africa

eatliveplay.dietitians@gmail.com

Background. Glutamine is considered essential during critical illness, and supplementation is common. Recent safety concerns highlight the need for glutamine research in specific populations.

Objective. To explore associations between plasma glutamine levels and clinical outcome in critically ill children.

Method. In this cross-sectional study, plasma glutamine was measured on admission and day 2 (D2) of paediatric intensive care unit (PICU) stay. Clinical and outcome data were prospectively captured over one month. A normal plasma glutamine was considered between 420 - 930 $\mu \mathrm{mol} / \mathrm{L}$.

Results. Of the 76 patients, (median (interquartile range (IQR)) age 19 months (3.6 - 64.8); 71\% male; median (IQR) PIM 3 - 4.23; IQR (-4.74 -3.21), most had normal plasma glutamine on admission (77.6\%; median (IQR) $556.5 \mu \mathrm{mol} / \mathrm{L}(459.5$ - 664.5)) and D2 (67.5\%; median (IQR) $529.0 \mu \mathrm{mol} / \mathrm{L}(356.0$ - 716.0)). Admission plasma glutamine varied significantly among diagnostic groups $(p=0.020)$, with trauma patients having the lowest values. Plasma glutamine was positively, but non-significantly, associated with length of hospital stay (LOS) $(r=0.23$; $p=0.067)$ and mortality risk $(r=0.22 ; p=0.052)$.

Conclusion. Among the patients, $78 \%$ had normal plasma glutamine levels at PICU admission. Although significant differences were identified among diagnostic groups, glutamine was not significantly related to LOS or mortality.
Prospective validation of a proposed severity-of-illness score in patients with tuberculosis requiring intensive care unit admission

U Lalla, E Irusen, B Allwood, J Taljaard, C F N Koegelenberg

Stellenbosch University and Tygerberg Academic Hospital, Cape Town, South Africa

usha@sun.ac.za

Background. We previously retrospectively validated a 6-point severityof-illness score identifying patients at risk of tuberculosis (TB) mortality in ICU. The parameters included septic shock, HIV infection with CD4 $<200$ cells/ $\mu \mathrm{L}$, renal dysfunction, $\mathrm{PaO}_{2}: \mathrm{FiO}_{2}<200 \mathrm{~mm} \mathrm{Hg}$, diffuse parenchymal infiltrates radiologically and no TB treatment on admission. Objective. To prospectively validate the severity-of-illness score.

Method. All patients admitted with TB over a 30-month period were included. A planned post-hoc analysis was performed to assess the significance of various parameters.

Results. Of 78 patients, $41(52.6 \%)$ died. The 6-point scores of nonsurvivors were higher $(3.5(1.3)$ v. $2.7(1.2) ; p=0.01)$. A score $\geq 3$ was associated with a higher mortality than $<3(64.0 \%$ v. $32.1 \%$; odds ratio (OR) 3.75; 95\% confidence interval (CI), 1.25 - 10.01; $p=0.01$ ). Post-hoc, a $\mathrm{PaO}_{2}: \mathrm{FiO}_{2}$ ratio $<200 \mathrm{~mm} \mathrm{Hg}$ and absence of $\mathrm{TB}$ treatment on admission failed to predict mortality, whereas any immunosuppression did predict mortality. A 4-point score (septic shock, any immunosuppression, renal dysfunction and lack of unilobar consolidation) was applied retrospectively. The 4-point scores of nonsurvivors were higher (3.4 (1.1) v. $2.2(1.1)$; $p<0.001)$. A score $\geq 2$ was associated with a higher mortality than a score $<2(66.7 \%$ v. $20.8 \%$; OR 7.60; 95\% CI 2.44 - 23.67; $p<0.001$ ).

Conclusion. The 6-point severity-of-illness score identified patients at higher risk of dying. We derived a simplified 4-point score with superior predictive power.

\section{Outcome of traumatic brain injury at a neurocritical care unit: A review of 189 patients}

A Oseni, ${ }^{1,2}$ C Arnold-Day, ${ }^{1,2} \mathrm{R}$ Lekoloane, ${ }^{1}$ J Lazarus, ${ }^{1}$ P Semple ${ }^{1,2}$

${ }^{1}$ Division of Neurosurgery, University of Cape Town, South Africa

${ }^{2}$ Division of Critical Care, University of Cape Town, South Africa

oseniabidemi@yahoo.com

Background. Traumatic brain injury (TBI) is a major public health concern, particularly in resource-constrained environments. Management focuses on neuroprotection and the prevention of both secondary brain injury and non-neurological complications.

Objective. To retrospectively analyse patients with TBI admitted into neurosurgical ICU in a tertiary hospital between January 2018 and December 2018.

Methods. Patients' basic demographics, mechanism of injury, admitting Glascow Coma Score (GCS), radiological findings and clinical diagnosis on ICU admission were reviewed. ICU length of stay, surgeries undergone, complications and adverse events during ICU course were also evaluated. The primary outcome was either survival or death.

Results. One hundred and eighty-nine patients ( $n=166 ; 87.8 \%$ male) with TBI were admitted into the ICU over the study period. The most common mechanism of injury was interpersonal assault, accounting for $40.4 \%$ ( $n=86)$ of cases, followed by road traffic accidents $(22.1 \%)$ and head gunshot wound (11.1\%). 
Overall, $13.1 \%$ of patients developed complications during the ICU stay, most commonly respiratory (5.2\%; including ventilator-associated pneumonia, lung collapse and pneumothoraxes), followed by sepsis (3.8\%). The crude ICU death rate among TBI patients was $6.4 \%$. Of the survivors, $59.7 \%$ were discharged to the step-down ward with Glascow Outcome Score $(\mathrm{GOS})>3$. There was significant correlation between admitting GCS and in-ICU mortality.

Conclusion. TBI continues to have significant morbidity and mortality; however, with dedicated neurosurgical intensive care focused on preventing secondary brain injury, the immediate short-term outcome appears to have improved.

\section{Recognise and rescue deteriorating patients: A large-scale quality improvement project to address failure to rescue}

\section{Y Walsh, ${ }^{1}$ A Prinsloo ${ }^{2}$}

${ }^{1}$ Mediclinic Southern Africa, Stellenbosch, Cape Town, South Africa

${ }^{2} \mathrm{Da}$ Vinci Institute of Technology, Johannesburg, South Africa

yolanda.walsh@mediclinic.co.za

Background. A colour-coded vital signs record with calling criteria for five physiological parameters is utilised in the general nursing units of a large hospital group in southern Africa. Data from nine medical units showed that only $23 \%$ of abnormal vital signs were reported for appropriate action to be initiated.

Objective. To ensure that $95 \%$ of abnormal vitals are managed appropriately in all general nursing units across a hospital group.

Method. Fifty-two hospitals dialled into separate monthly telephonic sessions ( 1.5 hours/session) and presented their data, progress and learning following an initial project briefing session. The monthly sessions were designed to teach each nursing manager, patient safety manager and unit manager how to apply improvement science to the problem for sustainable improvement.

Results. A total of $46(n=46 / 52)$ hospitals and 190 units completed the 9-month project. The project achieved a statistically significant reduction in the overall mortality index for the hospital group ( $n=52$ hospitals), from a pre-project baseline of 0.97 to 0.86 . This reduction has been sustained for 20 months.

Conclusion. The reliability of recognising and rescuing deteriorating patients can be improved with the effective management of abnormal vital signs - a critical foundational step in preventing failure to rescue.

\section{Using a massive open online course to teach the principles of deceased donation: Its place in the world D Thomson, ${ }^{1}$ F Waggie $^{2}$ \\ ${ }^{1}$ University of Cape Town, South Africa \\ ${ }^{2}$ University of the Western Cape, Cape Town, South Africa \\ thomson.david@gmail.com}

Background. Massive Open Online Courses (MOOCs) can educate on a large scale about best practices in deceased organ donation.

Objective. To describe the uptake, progression, demographics and perceptions of students who completed a MOOC on organ donation offered by an African university via a global platform.

Methods. A retrospective descriptive analysis was undertaken of participant survey, course completion and learner feedback data collected from all participants of the monthly 4-week course 'Organ Donation: From Death to Life' offered from July 2017 to December 2018.

Results. Of the 10601 unique views on the signup page, 2027 enrolled, and 1277 participants started the course. The largest number of participants were from South Africa (31.8\%), followed by the USA (18.1\%) and India (6\%). Most students were $<34$ years old $(73 \%)$, and women $(64 \%)$. Half of the participants $(50 \%)$ were not formally studying, and $23 \%$ were unemployed at the time of enrollment. A total of 381 participants (29.8\%) completed the course. Of the 81 ratings received, an average rating of 4.8 out of 5 was received.

Conclusion. Organ donor education projects using massive open online learning platforms offer an effective means to reach a potentially large and diverse audience.

\section{Factors influencing consent rates to deceased organ donation in the Western Cape, South Africa}

H Bookholane, ${ }^{1}$ A Michaelides, L Prins, ${ }^{2}$ L Human, ${ }^{2}$ L Steenkamp, F McCurdie, ${ }^{3}$ B Gili, ${ }^{4}$ D Thomson ${ }^{1,3}$

${ }^{1}$ University of Cape Town, South Africa

${ }^{2}$ Netcare Transplant Unit, Cape Town, South Africa

${ }^{3}$ Groote Schuur Hospital, Cape Town, South Africa

${ }^{4}$ Red Cross War Memorial Children's Hospital, Cape Town, South Africa

thomson.david@gmail.com

Background. South Africa has very low organ donation rates.

Objective. To describe the number of consent requests and factors influencing request.

Methods. Prospective descriptive study of all family requests for organ donation conducted in the Western Cape by the Groote Schuur, Red Cross War Memorial Children's Hospital (state) and Netcare (private) transplant coordinators for the period 1 May 2017 to 1 May 2018.

Results. The six coordinators ( 3 state and 3 private) recorded data on 83 consecutive family approaches from 16 hospitals. The number of family approaches was greater in the state $(n=74)$ compared with the private $(n=9)$ hospitals. The overall consent rate was $27.7 \%$ (state sector $24.3 \%$, private sector $55.5 \%$ ). More referrals came from trauma and emergency units $(n=55 ; 66.25 \%)$ than intensive care units $(n=25 ; 30.1 \%)$. Fluid resuscitation was required in $56(67.5 \%)$ potential donors. The main reason for consent refusal was 'against my religion' $(n=21)$ or 'culture' $(n=18)$.

Conclusion. Approaches for consent were low in the Western Cape (lower in the private sector), with a low consent rate (lower in the state sector). Donor management by treating clinical teams needs to be ongoing and active during the consent process.

\section{Mortality outcomes following admission to paediatric intensive care: A systematic review}

\section{Procter, ${ }^{1}$ B M Morrow, ${ }^{2}$ G Pienaar, ${ }^{3}$ M Shelton, ${ }^{2}$ A Argent ${ }^{1}$}

${ }^{1}$ Red Cross War Memorial Children's Hospital, Cape Town, South Africa

${ }^{2}$ Department of Paediatrics, University of Cape Town, South Africa

${ }^{3}$ Western Cape Department of Health, Cape Town, South Africa clairemprocter@gmail.com

Background. Paediatric intensive care has developed rapidly in recent years, with a dramatic increase in survival rates. However, concerns have been raised 
regarding the impact that paediatric intensive care unit (PICU) admission has on the child, with potential ongoing risk of death after discharge.

Objective. To describe the short- and long-term mortality outcomes of children following admission to a PICU.

Methods. A systematic literature search was conducted using predefined search strategies in PubMed/MEDLINE, Scopus (including EMBASE), Web of Science, EBSCO, ProQuest, Google Scholar and CENTRAL. All English-language studies reporting mortality outcomes of children under 18 years old (excluding neonates) admitted to a PICU were eligible for inclusion, regardless of study design. Outcomes measured were: short-term ( $<30$ days) and long-term ( $<3$ months, $<6$ months, $<1$ year, $<5$ years and $>5$ year) mortality following PICU discharge. Data were extracted and presented in a narrative synthesis, with graphical presentation where possible. Pooling of results was not possible owing to substantial heterogeneity.

Results. More than 20000 articles were initially identified, of which 50 were ultimately included. In-ICU mortality rates ranged from $1.3-50 \%$ in different settings. PICU mortality has reduced over time in high-income countries; however, this is not evident in low- and middle-income countries, despite some suggestion of improvements in specific units or regions. Studies from high-income countries consistently report standardised mortality rates $(\mathrm{SMR})<1$, indicating mortality rates lower than predicted mortality (based on admission risk of mortality scores), whereas SMR in low- and middle-income countries are more variable and frequently $>1$ (i.e. higher than expected mortality). Of the 13 studies that reported mortality after PICU discharge, the majority reported cumulative mortality rates that continue to rise up to 10 years post discharge. Children admitted to PICU continue to be at increased risk of death compared with children not admitted to PICU for many years following discharge.

Conclusion. Mortality in PICU is extremely variable, and outcomes appear worse in poorer-resourced environments. Children admitted to PICU are at risk of death during and after their admission. Further research is recommended to determine functional, psychosocial and health-related quality-of-life outcomes of PICU admission.

\section{Physical function outcomes of privately admitted intensive care unit patients at hospital discharge}

\section{H A Pool, A Lupton-Smith, S D Hanekom}

Division of Physiotherapy, Health and Rehabilitation Sciences, Faculty of Medicine and Health Sciences, Stellenbosch University, Cape Town, South Africa poolhelanie@yahoo.com

Background. Poor physical function following critical illness is a major contributor to morbidity in survivors. Physical function has not been described within the private healthcare population in South Africa.

objective. To describe muscle strength, physical function and exercise capacity in critical illness survivors at intensive care unit (ICU) and hospital discharge.

Methods. A prospective observational cohort study was conducted in the ICUs of a private hospital in the Western Cape Province.

Results. Preliminary results of 16 participants (8 male) with a mean (standard deviation (SD)) age of 68.19 (15.58) years are presented.
The mean (SD) severity of illness score (SAPS 3) was 59.13 (15.47). The median (interquartile range (IQR)) length of ventilation was 5.78 (3.93 - 9.67) days, mean (SD) total length of stay was 13.38 (7.85) days in ICU and 19.06 (11.1) days in hospital. Mean (SD) Medical Research Council sum score (MRC SS) was $47.82(8.13)$ at ICU discharge and median MRC SS of 53.00 (IQR 40 - 56) at hospital discharge. Walking distance (exercise capacity) was a median of 117 (IQR 87 - 120) metres. Mean (SD) physical function scores were 36.91 (6.06) at ICU discharge and 41.80 (5.47) at hospital discharge using the Chelsea Critical Care Assessment tool (CPAx).

Conclusion. Critical illness survivors demonstrated impaired physical function outcomes.

\section{An audit of endotracheal tube and tracheostomy cuff pressure monitoring in a surgical intensive care unit}

M Allam, ${ }^{1}$ C Augustyn, ${ }^{2}$ G Ramsay, ${ }^{3}$ N Ahmed ${ }^{2}$

${ }^{1}$ Medical School, University of Aberdeen, Scotland, UK

${ }^{2}$ Surgical Intensive Care Unit, Tygerberg Hospital, Cape Town, South Africa

${ }^{3}$ Department of General Surgery, Aberdeen Royal Infirmary, Scotland, UK nadiya@sun.ac.za

Background. Endotracheal (ET) and tracheostomy tubes have inflatable cuffs at the distal tip of the tube. When inflated, the seal with the wall of the trachea facilitates positive pressure ventilation and prevents the passage of gastric contents into the airways below. The ideal pressure range at which the cuff should be inflated is between 20 and $30 \mathrm{cmH}_{2} 0$. Excessive cuff pressures can lead to tracheal wall ischaemia, ulceration or rupture, and underinflated cuffs increase the risk of respiratory infections. Adequate monitoring and recording of cuff pressures is a relatively simple and inexpensive method of reducing these preventable complications.

Objective. To identify and improve on any deficiencies identified in ET and tracheostomy cuff pressure monitoring in a surgical intensive care unit (SICU) at Tygerberg Hospital, South Africa.

Methods. This was a two-cycle clinical audit. Intubated patients were identified daily in the SICU. A manometer was used to measure cuff pressure. Additional data were gathered from clinical notes. A total of 50 patients were identified for each audit cycle. Once the initial audit was completed, an intervention was implemented in the form of posters, presentations and increased accessibility to manometers within the SICU.

Results. Of the initial 50 tracheal cuff pressures measured, 16 (32\%) were within the recommended range, and 1 (2\%) set of notes contained a pressure reading. After intervention, a further 50 cuff pressures were measured as part of the audit loop. In the re-audit, the number of correctly inflated cuffs rose to $40(80 \%)$ and cuff pressures were recorded within 29 (58\%) sets of notes.

Conclusion. The standard of cuff pressure monitoring in this SICU improved considerably following this audit cycle. Further interventions in the form of improved documentation and nurse-led cuff pressure monitoring require consideration. 


\section{Knowledge, barriers and facilitators of early mobilisation of critically ill patients among nurses and physiotherapists \\ I Du Plessis, ${ }^{1} \mathrm{~N}$ Arnold, ${ }^{1} \mathrm{M}$ Hayden, ${ }^{1} \mathrm{M}$ Mashego, ${ }^{1} \mathrm{R}$ Masoga, \\ C Mkiva, ${ }^{1}$ P Monamola, ${ }^{1}$ K Soni, ${ }^{1}$ A Lupton-Smith ${ }^{1,2}$ \\ ${ }^{1}$ Division of Physiotherapy, Department of Health and Rehabilitation \\ Sciences, University of Cape Town, South Africa \\ ${ }^{2}$ Division of Physiotherapy, Department of Interdisciplinary Sciences, \\ Stellenbosch University, Cape Town, South Africa \\ aluptonsmith@sun.ac.za}

Background. Early mobilisation (EM) of critically ill patients improves short-term outcomes and may improve longer-term morbidity. Despite the benefits, implementation remains poor.

Objective. To investigate the knowledge of staff about and facilitators and barriers to EM among intensive care unit (ICU) staff.

Method. Self-administered questionnaires were completed by physiotherapists and nurses working in ICUs, over a 1-week period, at a tertiary hospital in Cape Town.

Results. Twenty-six nurses and 17 physiotherapists consented to participate. Physiotherapists and nurses were knowledgeable on EM with mean (standard deviation (SD)) scores of $85 \%$ (22.65) and 74\% (23.68), respectively. Both professions scored lowest in the safety knowledge domain, with mean (SD) scores of $70.68 \%(0.30)$ and $55.49 \%(0.24)$, respectively. Both physiotherapists (88.24\%) and nurses (73.08\%) reported lack of staff to be the greatest barrier to EM. Among the physiotherapists, $82 \%$ indicated that patients being too unwell was a barrier, and $69 \%$ of nurses indicated that patients being too sedated was a barrier. Interdisciplinary care was identified as essential by all staff for implementation of EM.

Conclusion. Modifiable knowledge gaps were identified. Identification of barriers and facilitators to EM are the first steps in improving EM practice within a unit.

\section{Physical outcome measures used for long-term follow-up after critical illness: A scoping review \\ I du Plessis, ${ }^{1}$ A Lupton-Smith, ${ }^{2}$ S Hanekom ${ }^{2}$ \\ ${ }^{1}$ Division of Physiotherapy, Department of Health and Rehabilitation \\ Sciences, University of Cape Town, South Africa \\ ${ }^{2}$ Division of Physiotherapy, Department of Interdisciplinary Sciences, Stellenbosch University, Cape Town, South Africa \\ ilse.duplessis@uct.ac.za}

Background. Reducing morbidity following critical illness is becoming increasingly important. Appropriate outcome measures (OM) are essential to quantify morbidity.

Objective. To map the available literature on physical OM used to describe physical function following critical illness.

Method. The review was conducted using the methodology detailed by the Joanna Briggs Institute (2015). EBSCOhost, Web of Science and Scopus were searched in March 2019. Publications reporting on physical function following critical illness were included. No time limits were applied. Two independent reviewers conducted the review.
Results. A total of 1078 publications were identified. Forty-one publications published between 1995 and March 2019 were included, 21 $(51.2 \%)$ were in the last 5 years. Nine $(21.9 \%)$ publications reported OM beyond 1 year post discharge. Twenty-nine different OM were identified and used at variable follow-up intervals. Ten (34.5\%) and 19 (65.5\%) OM measured at impairment and activity levels on the ICF, respectively. The Medical Research Council Sum Score $(n=26 ; 21.3 \%)$ and 6-minute walk test ( $n=42 ; 47.1 \%$ ) were used most frequently.

Conclusion. Publications have increased over the last 5 years. Limited publications measured beyond 1 year post discharge. OM addressing participation are lacking. The variability of $\mathrm{OM}$ emphasises the need for a core set of OM in the field.

\section{Why, how and when do children die in a paediatric intensive care unit in South Africa? \\ M Wege, ${ }^{1,2}$ B Morrow, ${ }^{1}$ B Rossouw, ${ }^{1,2}$ A C Argent ${ }^{1,2}$ \\ ${ }^{1}$ Department of Paediatrics and Child Health, University of Cape Town, South Africa \\ ${ }^{2}$ Red Cross War Memorial Children's Hospital, Cape Town, South Africa \\ martie.wege@gmail.com}

Background. Availability of paediatric intensive care (PICU) beds is a scarce resource in South Africa. Difficult decisions, such as limitation and withdrawal of life-sustaining therapy, are inevitable to optimise the use of PICU beds.

Objectives. To describe the characteristics and modes of death in a South African PICU.

Methods. Retrospective review of data extracted from the Child Healthcare Problem Identification Programme (Child PIP) and the PICU summary system on children of any age who died in the PICU between 1 January 2013 and 31 December 2017.

Results. There were 451 deaths reviewed (54\% male), with a median (interquartile range (IQR)) age of $7(1-30)$ months. The median (IQR) length of PICU stay prior to death was 3 ( 1 - 7) days. The mode of death in $23.7 \%$ ( $n=107)$ was withdrawal of life-sustaining therapies; $36.14 \%$ $(n=163)$ died after limitation of life-sustaining therapies; $22.0 \%(n=99)$ died after failed resuscitation; and $17.3 \%(n=78)$ were diagnosed brain dead. Ultimately, 60\% $(n=270)$ of children died after the decision was made to limit or withdraw life-sustaining therapies. Septicaemia (20.4\%) was the most common cause of death. Only $1.5 \%(n=7)$ of deaths became organ donors.

Conclusions. Most children died after a decision to limit or withdraw life-sustaining therapy.

\section{The indications and timing of haemodialysis in critically ill patients with acute kidney injury admitted to ICU.}

P M Brown, L Redford, S Omar

University of the Witwatersrand, Johannesburg, South Africa shahedicu@gmail.com 
Background. Wide variation in timing of initiation of renal replacement therapy (RRT) among clinicians has been observed. Currently, there are no national or Africa-specific guidelines for the timing of RRT initiation. Objective. To describe current practices in the timing of RRT in patients with AKI admitted to the intensive care unit (ICU) of an adult academic ICU.

Method. We performed a retrospective, descriptive study in an adult academic ICU in Soweto from 1 January 2013 to 31 December 2014.

Results. There were 2152 ICU admissions over 2 years. 76 (3.5\%) patients required renal replacement therapy (RRT). Sepsis was present in $83 \%$. The most common indication for RRT was oliguria/anuria in 38 (50\%) followed by worsening urea/creatinine (22; 29\%), metabolic acidosis (9; $11.8 \%)$, refractory hyperkalaemia $(4 ; 5.3 \%)$, fluid overload $(2 ; 2.6 \%)$ and other $(1 ; 1.3 \%)$. Forty-two patients $(55 \%)$ had RRT instituted on admission day (D0 RRT), and 34 (45\%) after D0. Ninety percent of D0 RRT and $94 \%$ of post-D0 RRT patients had KDIGO stage 3 AKI. Once a decision to initiate RRT was made, the median time to starting RRT was 4 hours. The composite outcome of death, RRT dependence and diuretic dependence at ICU was $21 \%$ (RRT initiated on D0 v. > D0; $p=0.22$.) The ICU mortality was $3 \%(n=2 / 76)$.

Conclusion. RRT was administered at KDIGO stage 3 in at least $90 \%$ of predominantly sepsis-related AKI, and was associated with a relatively low mortality.

\section{Paediatric cardiac mortality and palliative care in a developing country}

B Rossouw, ${ }^{1,2}$ M Meiring, ${ }^{2,3}$ B M Morrow, ${ }^{1,2}$ A C Argent ${ }^{1,2}$

${ }^{1}$ Division of Critical Care and Children's Heart Diseases, Red Cross War Memorial Children's Hospital

${ }^{2}$ Department of Paediatrics and Child Health, University of Cape Town

${ }^{3}$ PaedsPal Paediatric Palliative Care, Cape Town, South Africa

beyra.rossouw@uct.ac.za
Background. Paediatric cardiac mortality is higher in resourceconstrained than other countries, owing to limited access to advanced technology. The World Health Organization (WHO) recommends early palliative care (PC) for all those diagnosed with life-threatening or life-limiting conditions.

Objectives. To describe in-hospital mortality of children with known heart conditions, in a setting without mechanical cardiac support or transplantation available. Furthermore, the study aimed to describe PC referrals.

Method. A single-centre retrospective folder review conducted between February 2014 and May 2019.

Results. A total of 233 children were enrolled (median age 6 months). Diagnosis included acquired heart disease $(20 \%)$, cyanotic congenital heart disease (CHD) (40\%) and acyanotic CHD (40\%). Of the children with CHD, 53\% died after and $28 \%$ before planned cardiac surgery. Cardiac surgery was withheld in $19 \%$. Eleven children died of infective endocarditis. A total of $98 \%$ needed intensive care unit (ICU) care. Area of death was ICU, ward and emergency unit in $68 \%$, $31 \%$ and $9.5 \%$, respectively. Four children died in theatre. Terminal care included non-escalation of life support (43\%), withdrawal of life support (17\%) and failed resuscitation (39\%). Twenty-eight percent of children received palliative care services, at a median time of 9 days before their death.

Conclusion. Only a small number of children received PC at disease end-stage, contrary to the WHO's recommendation of early referral. 
36 SAJCC July 2019 , Vol. 35, No. 1 